\title{
Meditación y proceso de enseñanza aprendizaje: Una experiencia educativa con niñas y niños sordos
}

Octavio Poblete Christie. Universidad de Playa Ancha (Chile).

Ximena Vidal Mella. Universidad Mayor (Chile).

Verónica Gallardo Peña. Colegio San Francisco de Asís para niños sordos de Santiago de Chile.

Recepción: 29 de julio de 2016 | Revisión: 6 de septiembre de 2016 | Aceptado: 10 octubre de 2016 Correspondencia: Octavio Poblete Christie | Orcid ID: 0000-0003-2551-0878 | Email: octavio.poblete@upla.cl

Citar: Poblete, O., Vidal, X. y Gallardo, V. (2016). Meditación y proceso de enseñanza aprendizaje: Una experiencia educativa con niñas y niños sordos. ReiDoCrea, 5, 195-205.

Resumen: En este trabajo se expone una experiencia cuyo objetivo fue implementar, dentro de la rutina escolar, la práctica de meditación por parte de estudiantes y profesores, efectuando también, una primera aproximación evaluativa. La experiencia fue implementada en un colegio para niñas y niños sordos ubicado en la comuna de Santiago, Chile. Participaron 41 estudiantes de nivel básico y 11 profesores. Se trabajó con toda la comunidad educativa transitando por distintas etapas, desde un proceso de sensibilización hasta la ejecución definitiva y evaluación. Se utilizó respiración espontánea como base de la técnica meditativa. A un año de práctica con tres sesiones semanales se realizó una evaluación. A nivel cualitativo, los actores mostraron una apreciación positiva de la experiencia en distintos aspectos. Además, se diseñó y aplicó una escala Likert que midió la percepción docente del efecto de la práctica sobre el proceso enseñanza aprendizaje. Los resultados muestran una percepción favorable en todos los factores. Finalmente, se reflexiona sobre diversos aspectos de la experiencia, de la técnica y de la evaluación realizada.

Palabras clave: Meditación | Sordos

Meditation and the teaching-learning process. An educational experience with deaf children

Abstract: This article exposes an experience which aim was to implement student and teachers meditation practice within the school routine and developing as well a first assessment approach. The experience was implemented at a school for deaf children located at a commune in Santiago, Chile. Participants were 41 primary school children and 11 teachers. The work involved whole school community who went along different stages, from a process of sensitization to the final execution and its evaluation. Spontaneous breathing was used as base of the meditative technique. The evaluation was developed after one year of practice three times a week. From a qualitative perspective, participants showed a positive appreciation of the experience at different aspects. Moreover, a Likert scale was applied to measure teachers' perceptions about the effect of the practice on the teaching-learning process. Results show a favorable perception in all the factors. Finally, authors reflect about the diverse aspects of the experience, the technique and the evaluation.

Key words: Meditation | Deafness

\section{Introducción}

Existe amplio acuerdo respecto de la relevancia de las variables afectivas en el proceso de enseñanza aprendizaje (Coll, Palacios y Marchesi, 2001; Molina y Pérez, 2006; Madrigal, Díaz, Cuevas, Nova y Bravo, 2011). A su vez, de manera creciente se realizan declaraciones sobre la necesidad de incorporar en el currículum aspectos que potencien el desarrollo emocional de los alumnos/as (Casassus, 2006; Naranjo, 2007). El desafío para los educadores es sumamente complejo puesto que, pese a carecer de un entrenamiento que les permita fomentar tales aprendizajes en sus estudiantes ya que en su formación inicial no lo reciben (Canedo, 2014; Fernández, Palomero y Teruel, 2009; López-Goñi y Goñi, 2009), sí se les demanda avanzar en tales aspectos.

Por otra parte, durante las últimas décadas la meditación se ha venido popularizando. Tras una gran variedad de técnica meditativas disponibles descansa un mismo objetivo: la disminución de la actividad "mental" mediante el incremento de la atención y la percepción lo cual genera descanso en quien la ejercita (Sánchez, 2011). 
Los beneficios de esta práctica han sido reportados por la investigación (Tang, Hölzel y Posner, 2015). Existe evidencia, por ejemplo, de sus efectos positivos sobre la estructura y el funcionamiento cerebral, tales como incremento en la girificación (Luders, Kurth, Mayer, Toga, Narr y Gaser, 2012), activación de determinadas estructuras asociadas a la atención y las emociones, y cambios en las neuroplasticidad, (Bertolín, 2014). Se ha observado, fruto de su práctica, incremento de afectos de valencia positiva, aumento de atención y concentración, disminución de estrés negativo, sensación de seguridad de sí mismo, entro otros (Baptista y Dalbosco, 2009; Manocha et al. 2011; Campagne, 2004; Sánchez, 2011).

Similares efectos se ha reportado en diferentes contextos educativos. Estudiantes de primaria mejoraron sus resultados en matemática y lenguaje luego de incorporar la meditación a su Actividad Educativa (Nidich, Mjasiri, Nidich, Rainforth, Grant, Valosek, Chang y Zigler, 2011). Asimismo, en un trabajo con escolares adolescentes se constató un incremento de pensamientos positivos, reducción del estrés y alza de la atención en clases (Huppert y Johnson, 2010). En otra investigación similar, se observó mejora en el manejo del estrés, mayor conciencia de sí mismo, mayor atención, mejor estado de ánimo, mejor clima escolar y mayor participación en clases (Wisner, 2013). En el ámbito de la educación especial, un trabajo con 34 adolescentes con problemas de aprendizaje, mostraron disminución de la ansiedad y incremento en la atención (Beauchemin, Hutchins y Patterson, 2008). Efectos análogos se han observado en educadores y padres de niños con necesidades especiales (Benn, Akiva, Arel y Roeser, 2012) Finalmente cabe destacar una experiencia con estudiantes de pedagogía quienes reportaron, luego de un periodo de práctica de meditación, reducción del estrés percibido tanto dentro como fuera de su trabajo (Franco, 2009). Experiencias similares a las anteriores han sido documentadas también en otros países (Vaquera, 2006). Por lo mismo, la meditación también se visualiza como una herramienta importante para disminuir en estrés de profesores y mejorar el clima de aula (Csaszar y Buchanan, 2015).

Sin embargo, pese a estos antecedentes, la meditación aún no ha sido incorporada a los planes educativos de manera cabal en la mayor parte de los países occidentales, tal como ocurre en Chile. Lo anterior quizás podría explicarse por diversos factores, desde el desconocimiento o incluso prejuicios sobre esta disciplina, hasta el enorme sesgo cognitivo sobre el cual descansa nuestro sistema cultural y, por ende, nuestro sistema educativo.

El siguiente trabajo, constituye una experiencia piloto en el ámbito de la educación especial consistente en un proceso de tres años de trabajo, que permitió instalar la práctica de la meditación de alumnos y profesores en el quehacer cotidiano del establecimiento, lo cual, además, ha permitido tener una primera aproximación sobre sus efectos sobre el aprendizaje y el comportamiento escolar. Cabe destacar finalmente que la experiencia ha seguido progresando hasta el momento de esta publicación.

El objetivo de este trabajo es compartir los aspectos más relevantes que caracterizaron un proceso de implementación de la meditación en el aula como estrategia para favorecer el proceso de enseñanza aprendizaje en diferentes asignaturas del establecimiento. Se busca retratar tanto el proceso de implementación propiamente, el nivel de participación que ha generado la propuesta, así como la incidencia percibida de la meditación sobre el proceso de enseñanza aprendizaje todo lo cual permite, a su vez, reflexionar sobre lo realizado. 


\section{Descripción de la Experiencia}

En la experiencia participaron 11 docentes y 41 alumnos y alumnas de $1^{\circ}$ a $6^{\circ}$ básico y sus familias, de un colegio especial de niños de sordos ubicado en la comuna de Santiago de Chile. Lo estudiantes pertenecen a familias de un nivel socioeconómico medio bajo, en las que muchas veces se observan problemas de carácter psicosocial. Además de problemas auditivos, cerca de un $40 \%$ de los alumnos manifiesta además otras dificultades, principalmente SDA-H. El nivel de compromiso más común observado en los alumnos se ubica en los rangos hipoacusia Severa o Profunda. El colegio se caracteriza por un enfoque verbal auditivo y su proyecto considera un compromiso importante con el desarrollo socioemocional de sus estudiantes. El equipo que participó de la experiencia estaba compuesto por 8 profesoras de educación diferencial, 1 profesora de artes plásticas, 1 profesora de educación musical y 1 profesora de educación física.

Durante el año 2010, y como parte de la actividad de apoyo a la docencia en torno al desarrollo emocional (Barría, 2014), se realizó una introducción a la práctica de la meditación, proporcionando información sobre sus beneficios reportados científicamente. Además se realizaron sesiones que permitieron un acercamiento a la experiencia corporal, la autoconsciencia y a la práctica de meditación propiamente tal, fruto de lo cual se generó una disposición muy positiva a desarrollar aún más estos aspectos al interior del establecimiento, surgiendo la idea de proyectar este trabajo a toda la comunidad educativa. Siempre en los espacios del consejo de profesores se estableció un diálogo abierto en torno a esta idea lo cual permitió evaluar en conjunto la posibilidad de introducir a la rutina educativa la práctica de la meditación, considerando que podría ser un aporte para el desarrollo afectivo de los estudiantes; y que, por otra parte, los procesos de enseñanza aprendizaje del establecimiento frecuentemente se veían obstaculizados por dificultades atencionales y de inquietud motora y, otras veces, a factores afectivos posiblemente relacionados con la vida familiar de los estudiantes. Producto de todo esto, se diseñó una propuesta orientada a concretar esta iniciativa la cual se comenzó a implementar desde el año 2011, todo lo cual fue coordinado por el psicólogo del establecimiento, meditante activo y entrenado en diversas técnicas meditativas.

El proceso de implementación de la práctica se realizó en tres etapas: difusión del proyecto a la comunidad educativa, práctica piloto y retroalimentación, e implementación definitiva.

Difusión: Se comenzó por difundir y sensibilizar a la comunidad educativa de las características de la meditación. Con las docentes se realizaron dos reuniones de trabajo. Una para dar a conocer la propuesta, resolver inquietudes y ajustarla en función de observaciones surgidas por el equipo. En la segunda reunión, se analizó un capítulo del programa "Redes" de la televisión española sobre el tema, lo cual permitió motivar el trabajo y conectarlo con el quehacer educativo. Además se entregó material de apoyo (enlaces, textos, etc.), y se orientó la propuesta del trabajo de sensibilización a realizar con niños y apoderados.

Posteriormente, durante las reuniones con padres y apoderados, las profesoras pudieron despejar dudas y derribar algunos mitos existentes, como por ejemplo, la supuesta conexión entre la meditación y la adopción de algún credo religioso. Además, realizaron una breve con orientación de las profesoras lo cual les permitió familiarizarse con la técnica seleccionada. 
El trabajo de difusión con alumnos estuvo a cargo de los profesores jefes respectivos y fue realizado en horarios de clases. Para ello se implementó un breve taller donde se conversó de estos temas y se realizaron ejercicios de relajación y de atención al propio cuerpo y respiración. Así mismo se analizó en conjunto un breve video disponible en internet sobre meditación y que resultó de mucha utilidad. También se realizaron algunas actividades destinada a representar plásticamente la meditación cuyos resultados fueron expuestos posteriormente a toda la comunidad.

Finalmente, se instaló en los espacios comunes del colegio, diversos paneles con imágenes y textos que informaban sobre diversos aspectos relacionados con la meditación así como su conexión con valores de tradición cristiana que el Colegio busca incentivar, tales como "paz", la "fraternidad" o "ecuanimidad". Asimismo, se incorporó el uso de mandalas como elemento decorativo y como material pedagógico.

Durante el transcurso de todas estas actividades se mantuvo un proceso de constante retroalimentación entre profesores y el coordinador del proyecto, lo que permitió ir analizando diversos aspectos tanto técnicos como operativos que pudieran obstaculizar o bien favorecer el avance de la experiencia. Esta labor se realizó vía correo electrónico y, en su última etapa, a través de reuniones presenciales todo lo cual permitió planificar con detalle el trabajo a realizar con los alumnos y alumnas.

Práctica piloto: Esta etapa permitió diseñar los espacios y tiempos concretos en que cada grupo dedicarían a la práctica. De esta forma, se obtuvo como producto un listado de los horarios de meditación para cada uno de ellos, el diseño y elaboración de una señalética especial para cada sala.

Luego de una revisión por los miembros del equipo se determinó que la técnica de referencia a utilizar con los alumnos y alumnas sería el uso de la respiración, contando exhaladas hasta el número diez para volver a uno nuevamente y ante el surgimiento de imágenes o pensamientos, regresar a la atención a la respiración. Esta modalidad fue la seleccionada dentro de otras posibles por estimarse como la más apropiada puesto que permitía apoyarse en estímulos concretos. Asimismo se diseñaron diversos tipos de apoyo para ajustar el entrenamiento a las necesidades educativas especiales de los alumnos.

En el mes de noviembre, se realizó el entrenamiento a los alumnos/as exigiendo un alto nivel de creatividad a los profesores para explicar en detalle la técnica meditativa. Luego, durante dos semanas se implementó una secuencia de tres prácticas por semana con cada curso lo cual permitió ajustar aspectos como los horarios, tiempos de prácticas, coordinación operativa, preparación del lugar, etc. Los tiempos destinados variaron por cada grupo dentro de un rango de 5 a 10 minutos. El momento en que se meditó en el transcurso de una clase varió también de grupo en grupo. Algunos optaron por meditar al principio de la clase, muchas veces luego de un recreo, lo cual permitió disminuir el nivel de agitación con el que regresaban de éste. Otros grupos optaron por hacerlo al final de la clase, como una forma de terminar de manera armónica el trabajo de la asignatura. Finalmente, otros lo hicieron a mitad de la sesión como una forma de abordar el cansancio e inyectar energía a la actividad. También, y de manera espontánea, la meditación fue utilizada eventualmente y con gran utilidad ante problemas emergentes de convivencia en sala.

Durante esta fase se pudo ir guiando el adecuado aprendizaje requerido para ejecutar la técnica. Acá se pudo ir observando que algunos niños/as reportaban haber notado que al comenzar la práctica solían imaginar o evocar recuerdos ante lo cual redirigían efectivamente su atención a la respiración. Otros en cambio, se mostraron 
excesivamente atentos al conteo mecánico de la respiración perdiendo con ello el objetivo de la práctica todo lo cual generó la necesidad de proponerles como variante prestar atención sólo a la respiración, sin contar, lo cual contribuyó a mejorar significativamente su ejecución.

Asimismo, se verificó además la disposición espacial de los alumnos en el aula a fin de evitar la continuidad pedagógica de la clase así como eventuales interrupciones durante la práctica. Algunos cursos meditaron en sus mismas sillas y otros sobre cojines dispuestos en el piso de la sala. No obstante, mayoritariamente los diferentes estudiantes optaron por meditar en sus propios puestos. Se implementó el uso de distintivos en las puertas de las salas durante la práctica a fin de evitar interrupciones externas. Además, se fue experimentando estrategias alternativas con los niños más inquietos o menos motivados. En el caso de los niños más inquietos, se les ofreció mantener los ojos abiertos e incluso realizar una actividad alternativa en silencio, como dibujar, pintar, contemplar una vela, entretenerse de manera silenciosa con algún juguete, entre otras.

Una de las principales adaptaciones a la técnica de meditación utilizada a fin hacerla más amigable con las características de los estudiantes estuvo destinada a la manera de informarles el inicio y término de la práctica. Para ello, se recurrió al contacto a través de la mano y "en cadena" entre ellos lo cual permitió adicionalmente disminuir la ansiedad que en algunos ocasionaba el hecho de mantener los ojos cerrados. También fue destacable observar cómo algunos alumnos con dificultades motoras (hipotonías), fueron elevando su conciencia y control corporal cuando mantenían los ojos cerrados. En general, notoria la tranquilidad observada en los alumnos al finalizar cada práctica lo cual, además, fue explícitamente reportado por ellos. La mayor parte de los niños y niñas, mostraban experiencias de relajo intenso, lo cual se podía visualizar también en los estiramientos espontáneos de su cuerpo una vez finalizada cada sesión. Un alumno, por ejemplo, gesticulando la forma de una esfera con sus manos, reportaba la sensación gratificante experimentada al término de práctica, queriendo informar que lo había dejado "redondo". Otra, mientras hacía referencia a su corazón con una mano, desplazaba suavemente la otra en sentido horizontal, indicando con ello que ahora se sentía más tranquila.

Retroalimentación: Terminado el período de práctica piloto, el coordinador del proyecto solicitó retroalimentación a todos los actores. Los alumnos, la entregaron directamente a sus profesores tanto a través del diálogo directo como a través de dibujos. Las profesoras informaron que mayoritariamente los estudiantes encontraron que la actividad les resultó muy atractiva lo cual se observaba, además, en que ellos mismos les recordaban los horarios pese a que en ocasiones ellas olvidaban; o bien, solicitaban sesiones de meditación adicional previamente a la realización de algunas prueba de asignatura a fin de tranquilizarse. En esta etapa destacó el reporte de una alumna quien relató recomendar a su madre meditar cuando la notaba nerviosa en casa, adoptando ella comportamientos análogos como sentarse en un cojín o presentarle velas para encender.

Asimismo, las profesoras respondieron la siguiente pregunta: “¿Lograron meditar de acuerdo a los tiempos estipulado con cada grupo y qué factores incidieron sobre la experiencia?".

En general, las profesoras indicaron que los resultados fueron satisfactorios, lo cual se vio reflejado en que la mayor parte de los grupos meditó las veces acordadas; los estudiantes adoptaron una actividad muy positiva por la práctica; los propios niños y niñas espontáneamente recordaban a los profesores realizar la actividad; los grupos 
que comenzaron con menos minutos gradualmente fueron solicitando incrementar el tiempo de meditación; los estudiantes fácilmente adoptaron una postura corporal acorde; las mínimas situaciones disruptivas observadas al inicio disminuyeron gradualmente, etc. Asimismo, las profesoras destacaron un aumento en la habilidad de atención y concentración en clases luego de la práctica, así como una baja en los niveles de ansiedad. Fruto de todo lo anterior, al finalizar esta etapa afloró espontáneamente la solicitud por proseguir a futuro con este trabajo y aumentar los tiempos de meditación.

Por último, también se consultó en la reunión de mes a los apoderados quienes también confirmaron el alto impacto de esta actividad en los niños y niñas, lo cual pudieron observar en reportes sobre ésta, intentos y meditación espontánea en el hogar, invitación a ellos a meditar, etc.

Implementación definitiva: Considerando los resultados obtenidos se acordó con el cuerpo docente implementar la práctica en el Establecimiento durante el siguiente año de manera permanente, manteniendo los tres días a la semana para todos los cursos a partir de primero básico. También se sugirió continuar con actividades de apresto a la meditación en cursos menores. Se definió como tiempo máximo los 10 minutos.

De esta forma, y a partir de marzo de ese año todos los grupos realizaron práctica de meditación tres veces por semana. En general, la disposición positiva se mantuvo e incluso se fue reforzando incorporándose rápidamente al quehacer y en cierta forma, a la cultura del Establecimiento.

La experiencia aún prosigue al momento de presentar este trabajo, iniciando ya su cuarto año. En general, el formato se ha mantenido, aunque en el caso de los alumnos y alumnas más grandes se ha incursionado con un tiempo mayor de práctica y también se ha comenzado a explorar un trabajo de apresto a la meditación en niveles preescolares. Además, se han ido agregando otras actividades adicionales tales como la meditación colectiva en algunos hitos del año, en la que participan todas las profesoras y todos los alumnos y alumnas; y la meditación también con apoderados e invitados.

\section{Medición final}

Como producto de los reportes obtenidos de las profesoras sobre su percepción del efecto de la meditación tanto en sus propios comportamientos como en sus respectivos estudiantes, en relación el proceso de enseñanza aprendizaje, se diseñó un instrumento que buscó recopilar esta información de manera más ordenada.

Este instrumento permitía evaluar en una escala de 1 a 10 los beneficios percibidos por los profesores acerca de la práctica sobre diversos aspectos del proceso de enseñanza aprendizaje. La escala tenía dos formatos: una orientada a conocer la percepción sobre el propio comportamiento de las profesoras y otra, orientada a conocer la percepción sobre el comportamiento de los alumnos. En general se trató de las mismas dimensiones utilizadas para ambos formatos aunque con algunas diferencias. Estos factores o dimensiones fueron las siguientes:

Atención y concentración: Nivel atencional y de concentración durante la actividad educativa, una vez realizada la práctica de meditación.

Disciplina y comportamiento escolar: Aspectos normativos durante la clase y posterior a la práctica de meditación. En el caso de profesores se relaciona con la habilidad 
para propiciar una conducta de sus alumnos acorde al proceso de enseñanza aprendizaje; y en el caso de los alumnos, disposición a desplegar una conducta normativa, luego de la práctica de meditación.

Motivación: Motivación por la actividad educativa tanto en profesores como alumnos una vez de realizada la práctica.

Proceso enseñanza aprendizaje: Habilidad percibida en los propios profesores para desplegar estrategias de enseñanza y, en el caso de los alumnos, habilidad para avanzar hacia los objetivos de aprendizajes de la sesión.

Logro de aprendizajes: Percepción de los aprendizajes logrados por los alumnos. (Sólo alumnos).

Ansiedad: Disminución del nivel de ansiedad tanto en profesores como alumnos una vez de realizada la práctica.

Efecto global: Efectos positivos globales percibidos de la práctica de la meditación sobre la práctica pedagógica, en el caso de los profesores; y sobre la conducta educativa, en el caso de los alumnos.

El siguiente es un ejemplo del tipo de reactivo utilizado:

"Una vez realizada la meditación, mi propio nivel de atención y concentración en clases":

\begin{tabular}{|c|c|c|c|c|c|c|c|c|c|}
\hline \multicolumn{6}{|c|}{ Disminuyó } & \multicolumn{4}{|c|}{ Aumentó } \\
\hline Signifi & var & & & & & & \multicolumn{3}{|c|}{ Significativamente } \\
\hline 1 & 2 & 3 & 4 & 5 & 6 & 7 & 8 & 9 & 10 \\
\hline
\end{tabular}

El instrumento fue contestado por diez de profesoras que participaron de la experiencia. A continuación se exponen los resultados para ambas formas:

\section{Resultados}

\section{a) Profesores}

La figura 1 muestra las medias y la desviaciones estándar para cada una de las dimensiones en los propios profesores. 
Figura 1. Factores Profesores

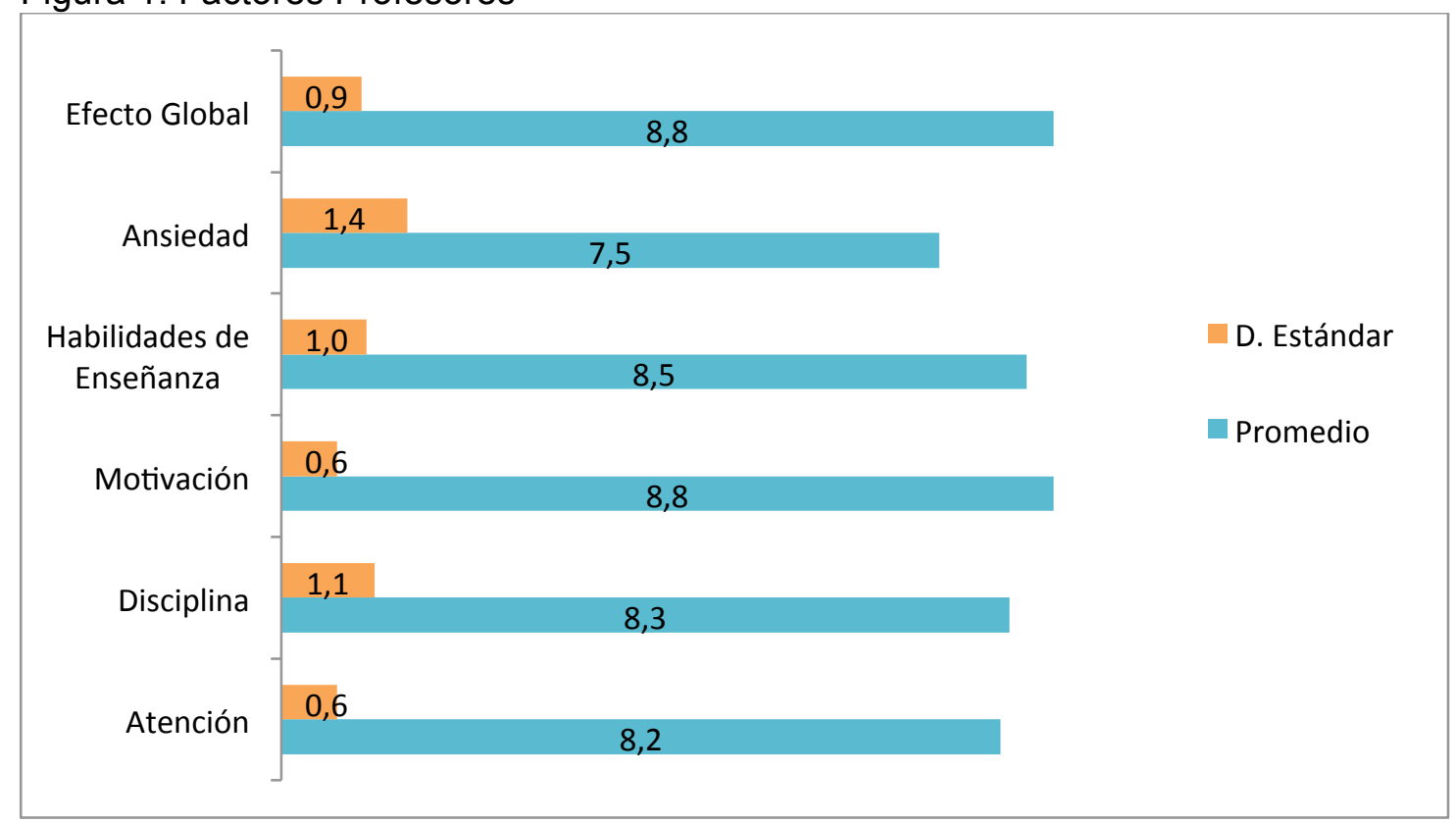

Cinco de las seis dimensiones obtuvieron una media mayor a 8 lo cual se puede considerar alto. Finalmente, es destacable que la desviación mayor se observó en "Ansiedad", variable que obtuvo además la media más baja, lo que se podría explicar por el peso de las diferencias individuales sobre los beneficios de la práctica de meditación.

\section{b) Alumnos}

La figura 2 muestra las medias y desviaciones estándar para cada una de las dimensiones en los estudiantes.

Figura 2. Factores alumnos

\begin{tabular}{|c|c|c|}
\hline Efecto Global & 1,1 & \\
\hline Ansiedad & 0,9 & \\
\hline Aprendizajes & 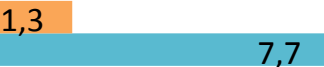 & \\
\hline Disposición & 1,2 & D. Estándar \\
\hline Motivación & 1,1 & \\
\hline Disciplina & 1,4 & \\
\hline Atención & 7,7 & \\
\hline
\end{tabular}


Destaca el alto nivel percibido del efecto global de la meditación sobre el comportamiento escolar. En el análisis por factor, destaca "Motivación" y "Ansiedad". Sobre estas dos últimas dimensiones se observan además mayor acuerdo entre los docentes. Finalmente, llama la atención el alto valor observado en las medias de profesores y alumnos el "Efecto global" lo cual reflejaría una apreciación general positiva de la actividad para el quehacer escolar. Por otra parte, destaca la diferencia observada en las medias entre ambos actores en la variable "Ansiedad", lo cual se podría explicar por la labor de dirección que implica para las docentes llevar adelante esta experiencia.

\section{Discusión}

Uno de los factores que colaboró sustantivamente al éxito de la experiencia fue la involucración gradual de los diferentes niveles del establecimiento. Se trabajó en "cascada", comenzando desde los directivos y profesores, pasando luego a los apoderados, para finalizar en los estudiantes. Además, se entregó a los docentes información de carácter científica sobre el tema y se realizó con ellos un acercamiento experiencial.

Como consecuencia de lo anterior, los estudiantes comenzaron a meditar al interior de una comunidad familiarizada y comprometida con esta práctica. Cada paso consideró retroalimentación en diferentes niveles todo lo cual fue de gran utilidad pues permitió ir abordando oportunamente cada uno de los detalles que implicaba un proceso de este tipo.

La iniciativa fue diseñada en función de las características de propias de los estudiantes. El trabajo de difusión y sensibilización fue cuidadoso, de tal forma que les permitiera a ellos comprender cabalmente la técnica. Durante todo el proceso se estuvo incorporando diversos apoyos necesarios, como lecturas, uso de la gestualidad, videos, dibujos, etc.

Por otra parte, la práctica no interfirió sobre la rutina escolar. La meditación fue practicada antes, durante y después de clases, a primeras horas del día y en momentos más cercanos a la tarde. Se trabajó con diferentes lapsos de tiempo y según diferentes disposiciones espaciales.

El esfuerzo realizado permitió instalar una técnica extraída de un linaje cultural cuyos supuestos epistemológicos son diferentes al que cultiva el currículum tradicional. El modelo educativo regular apunta principalmente al fomento de operaciones mentales de naturaleza simbólica, propias de la mente teórica (Pozo, 2011), buscando generar o compartir representaciones de una "realidad". En contraposición, la meditación pone en el centro la actividad autoconsciente y experiencial del propio observador, más allá de los contenidos o representaciones que su mente de pueda construir. Se trata además de un ejercicio de naturaleza esencialmente perceptiva y fenomenológica cuya práctica provoca sensaciones de bienestar, todo lo cual redunda en una mejor disposición para relacionarse con el entorno. De esta forma, mientras el currículum tradicional cultiva principalmente el pensamiento y reflexión, la meditación pone su acento en el sentir. Sin embargo, pese a que esta división de la actividad mental entre pensar y sentir resulta artificial (Damasio, 1999), el sistema educativo occidental aún no ofrece una propuesta que se ajuste a estos hallazgos.

En este sentido, los resultados de esta experiencia son alentadores puesto que su ejecución permite incorporar al trabajo educativo una dimensión de lo mental que suele quedar marginada, básicamente debido a la ausencia de metodologías 
apropiadas. Asimismo, resulta de interés indagar con mayor profundidad sobre el efecto que esta técnica experiencial podría tener sobre el proceso de enseñanza aprendizaje así como sobre las relaciones interpersonales que le dan vida a toda una comunidad educativa, particularmente en aquellas comunidades donde existen estudiantes con necesidades educativas especiales.

De todas formas, a la luz de esta experiencia, es posible afirmar que la meditación surge como un apoyo concreto que el espacio educativo puede ofrecer a toda la comunidad. No obstante lo anterior, es importante considerar la necesidad de ir desarrollando metodologías, técnicas e indicadores más precisos que permitan evaluar los avances de este tipo de trabajo sobre las diversas aristas del trabajo educativo.

\section{Referencias}

Barría, X. (2014). Colegio San Francisco de Asís para niños sordos, en John Miller, Mihele Irwin \& Kelly Nigh (2014). Teaching from the Thinking Heart, The Practice of Holistic Education (43-54). Washington: Information Age Publishing.

Baptista C. \& Dalbosco, D. (2009). Os Efeitos da Meditação à Luz da Investigação Científica em Psicologia: Revisão de Literatura. Psicologia ciência e profissão, 29 (2), 276-289.

Beauchemin, J., Hutchins, T. \& Patterson, F. (2008). Mindfulness Meditation May Lessen Anxiety, Promote Social Skills, and Improve Academic Performance Among Adolescents With Learning Disabilities, Journal of Evidence-Based Complementary \& Alternative Medicine, January 2008 vol. 13 no. 1 34-45.

Benn, R., Akiva, T., Arel, S. \& Roeser, R. (2012). Mindfulness training effects for parents and educators of children with special needs. Developmental Psychology, Vol 48(5), Sep, 1476-1487. doi: 10.1037/a0027537.

Bertolín, J. (2014). Sustratos psiconeurobiológicos de la meditación y la conciencia plena. Psiquiatría Biológica, Volume 21, Issue 2, May-August 2014, Pages 59-64

Campagne, D. (2004). Teoría y fisiología de la meditación. Cuadernos de Medicina Psicosomática y Psiquiatría de enlace, $\mathrm{N}^{\circ} 69 / 70,15-30$.

Canedo, D., (2014). Conocimiento y habilidades relativas a convivencia escolar presentes en la formación inicial docente. Memoria para optar al título de psicóloga, U. de Chile.

Cassasus, J. (2006). La educación del ser emocional. Santiago de Chile: Cuarto Propio/Índigo.

Coll, C., Palacios, J. y Marchesi, A. (2001). Desarrollo psicológico y educación: Psicología de la educación escolar. Madrid: Alianz.

Csaszar I., \& Buchanan, T. (2015). Meditation and Teacher Stress. Dimension of Early Childhood, Vol 43, number 1, 2015.

Damasio, A. (1999). El error de descartes, la razón de las emociones. Santiago: Andrés Bello.

Fernández, M., Palomero, J. y Teruel, M. (2009). El desarrollo socioafectivo en la formación inicial de los maestros, Revista Electrónica Interuniversitaria de Formación del Profesorado, 12 (1), 33-50.

Franco, J. (2009). Reducción de la percepción del estrés en estudiantes de Magisterio mediante la práctica de la meditación fluir. Apuntes de Psicología, Vol. 27, N 1, p.99-109.

Huppert, F. \& Johnson, D. (2010). A controlled trial of mindfulness training in schools; the importance of practice for an impact on well-being. The Journal of Positive Psychology. July 2010, Vol 5, Issue 4, 264-274.

López-Goñi, I. y Goñi Zabala, J. (2009). La competencia emocional en los currículos de formación inicial de los docentes. Un estudio comparativo. Revista de Educación, 357. Enero-abril 2012, pp. 467-489.

Luders E., Kurth F., Mayer E., Toga, A., Narr K. \& Gaser, C. (2012). The unique brain anatomy of meditation practitioners: alterations in cortical gyrification. Front. Hum. Neurosci. 6:34. doi: 10.3389/fnhum.2012.00034 
Manocha, R., Black, D., Sarris, J. \& Stoug, C. (2011). A Randomized, Controlled Trial of Meditation for Work Stress, Anxiety and Depressed Mood in Full-Time Workers. Evidence-Based Complementary and Alternative Medicine, Vol. 2011, Article ID 960583, 8 pages, doi:10.1155/2011/960583.

Molina, N. \& Pérez, I. (2006). El clima de relaciones interpersonales en el aula un caso de estudio. Paradigma, Vol. 27, N. 2, Maracay, Diciembre.

Madrigal, C., Díaz, A., Cuevas, C., Nova \& C., Bravo (2011). Clima social escolar en el aula y vínculo profesor-alumno: alcances, herramientas de evaluación, y programas de intervención. Revista Electrónica de Psicología Iztacala. 14, (3).

Naranjo, C. (2007). Cambiar la educación para cambiar el mundo. Santiago: Espacio Índigo.

Nidich, S., Mjasiri, S., Nidich, R., Rainforth, M., Grant, J., Valosek, L., Chang, W. \& Zigler, R. (2011). Academic Achievement and Transcendental Meditation: A Study with At-Risk Urban Middle School Students, Education, Vol. 131, No. 3.

Pozo, J. (2011). Aprendices y maestros: La psicología cognitiva del aprendizaje. Madrid: Alianza.

Sánchez, G. (2011). Meditación, mindfulness y sus efectos biopsicosociales. Revisión de literatura. Revista Electrónica de Psicología Iztacala. Junio, 14, (2). ISSN: 1870-8420

Rosaen, C. \& Benn, R. (2006). The Experience of Transcendental Meditation in Middle School Students: A Qualitative Report. The Journal of Science and Healing, Vol. 2, Issue 5, Pages 422-425, September.

Tang, Y., Hölzel, B. \& Posner, M. (2015). The neuroscience of mindfulness meditation, Nature Reviews Neuroscience, 16, 213-225, (2015), doi:10.1038/nrn3916.

Vaquera, E. (2006). Relajación, Meditación y Autoconocimiento Mental y Emocional para Docentes. Revista Digital Práctica docente, N³ (julio/septiembre. 2006), CEP de Granada. ISSN: 1885-6667. DI: gr-2475/05.

Vargas, M. (2010). La Meditación y la Relajación en la Educación. Hipnológica, 3:22-23. Julio/Agosto.

Wisner, B. (2013). An Exploratory Study of Mindfulness Meditation for Alternative School Students: Perceived Benefits for Improving School Climate and Student Functioning. Mindfulness, Vol 5, Issue 6, pp. 626-638.

\section{Fuentes electrónicas}

PROGRAMA “REDES”, RADIOTELEVISIÓN ESPAÑOLA (RTVE), Capítulo 50, "Meditación y aprendizaje”. Recuperado el 4 de febrero de 2016, (parte 1). https://www.youtube.com/watch?v=dcbASVA2E2U

PROGRAMA “REDES”, RADIOTELEVISIÓN ESPAÑOLA (RTVE), Capítulo 50, "Meditación y aprendizaje”. Recuperado el 4 de febrero de 2016, (parte 2). https://www.youtube.com/watch?v=UcJ2EbSxuyc

PROGRAMA “REDES”, RADIOTELEVISIÓN ESPAÑOLA (RTVE), Capítulo 50, “Meditación y aprendizaje”. Recuperado el 4 de febrero de 2016, (parte 3). https://www.youtube.com/watch?v=9dDwG_-z8P4

COMO MEDITAR EN UN MINUTO. Recuperado el 4 de febrero de 2016, de 